\title{
AVALIAÇÃO DAS CONDIÇÕES HIGIÊNICAS DAS MASSAS ALIMENTÍCIAS (MACARRÃO) , POR MICROSCOPIA ANALÍTICA
}

\author{
CARLOS HENRRY BELLOT VARGAS*
}

ARMANDO ANTUNES DE ALMEIDA*

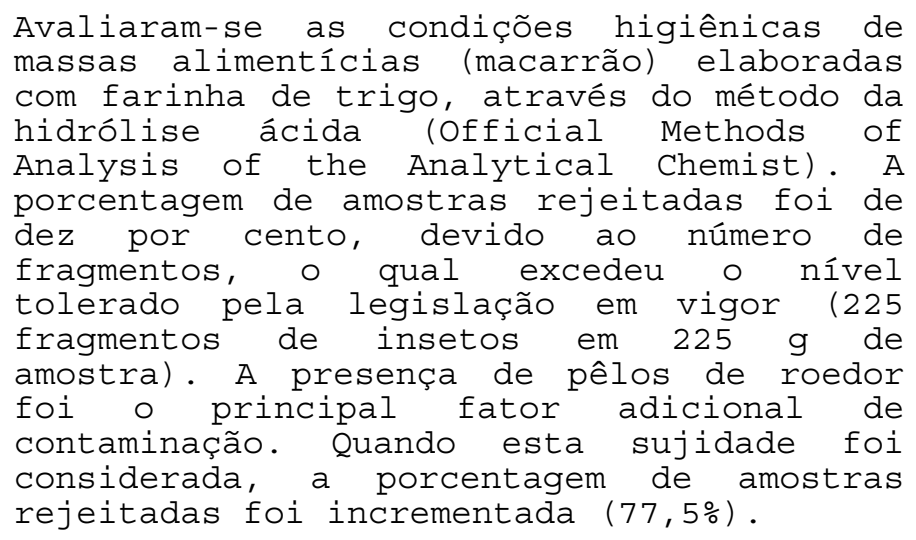

\section{INTRODUÇÃO}

A principal matéria-prima das massas alimentícias (macarrão) é a farinha de trigo, a qual pode apresentar contaminação biológica. Os principais contaminantes encontrados são os fragmentos de insetos, resultantes da moagem de grãos de trigo que apresentam infestação interna ou externa.

As infestações podem ocorrer no campo, no armazém e/ou nos moinhos. No caso das massas alimentícias, sua contaminação decorre, principalmente da utilização de farinhas contaminadas e/ou infestadas durante a estocagem, sob condições higiênicas não satisfatórias. Estes alimentos estão, também, sujeitos a contaminação adicional, durante sua elaboração e armazenamento, pelos materiais usados para empacotamento na indústria de pastifício, nos depósitos e/ou estocagem dos estabelecimentos comerciais.

Outras sujidades, como pêlos de roedor e ácaros podem também contaminar estes produtos. Segundo ZAMBONI \& ATUI (7) os pêlos de roedor são provenientes dos excrementos deixados por ratos ou camundongos no trigo ou na farinha estocada.

* Bolsistas do CNPq, Departamento de Zoologia, Universidade Federal do Paraná. 
A contaminação por ácaros, neste produto, está relacionada com condições favoráveis ao seu desenvolvimento. Se o armazenamento do trigo, da farinha e/ou do macarrão for inadequado, isto é, em ambiente com umidade relativa superior a $68 \%$ e temperaturas elevadas, e não houver limpeza do ambiente, os gêneros alimentícios estarão sujeitos a este tipo de contaminação (7).

LANDIN (6) avaliando as condições higiênicas de massas alimentícias, tipo espaguete, comercializadas em diferentes Estados do País (Minas Gerais, Rio de Janeiro, Rio Grande do Sul e São Paulo), observou que 76\% das amostras apresentaramse em desacordo com a legislação em vigor.

ZAMBONI \& ATUI (7) analisando 150 amostras de macarrão de três tipos, comercializadas na Capital e no Interior do Estado de São Paulo, perceberam que 48\% das amostras de macarrão com ovos, $80 \%$ das de macarrão comum e 58\% das de macarrão de sêmola, estavam fora dos padrões estabelecidos pela legislação vigente. Deve-se salientar que os autores citados fundamentaram os índices de condenação de acordo com a legislação em vigor na época, a qual tolerava até 30 fragmentos de insetos em $100 \mathrm{~g}$ de amostra e ausência de outras sujidades.

A legislação em vigor tolera até 225 fragmentos de insetos em $225 \mathrm{~g}$ de amostra de massas alimentícias e exige ausência de pêlos de roedor, ácaros vivos e/ou mortos, larvas e/ou insetos vivos e/ou mortos (2).

Este trabalho teve por objetivo verificar as condições higiênicas das massas alimentícias (macarrão), produzidas por quatro indústrias de pastifício e consumidas na cidade de Curitiba-PR.

\section{MATERIAL E MÉTODOS}

Foram analisadas 40 amostras de massas alimentícias (macarrão), de um kilo cada uma, elaboradas com farinha de trigo, produzidas e comercializadas durante o período de março a dezembro de 1990, por três indústrias de pastificio, localizadas no município de Curitiba e uma em São José dos Pinhais (PR). Utilizou-se o método da hidrólise ácida para determinação de sujidades leves em massas alimentícias, conforme descrito no official Methods of Analysis of the Association of Official Analytical Chemists (1). Cada amostra foi analisada em triplicata, perfazendo 120 análises.

\section{RESULTADOS E DISCUSSÃO}

Os resultados obtidos na pesquisa de sujidades leves através do método da hidrólise ácida estão apresentados nas Tabelas 1 e 2 . 
TABELA 1 - FRAGMENTOS DE INSETOS, INSETOS MORTOS, ÁCAROS E PÊLOS DE ROEDOR ENCONTRADOS NAS AMOSTRAS DE MACARRÃO, PRODUZIDAS E COMERCIALIZADAS POR QUATRO INDÚSTRIAS DE PASTIFÍCIO, LOCALIZADAS NO ESTADO DO PARANÁ, ANALISADAS ATRAVÉS DO MÉTODO DA HIDRÓLISE ÁCIDA

\begin{tabular}{|c|c|c|c|c|c|c|c|c|}
\hline \multirow{2}{*}{$\begin{array}{l}\text { Amostra } \\
\text { Sujidades }\end{array}$} & \multicolumn{2}{|c|}{ Marca A } & \multicolumn{2}{|c|}{ Marca B } & \multicolumn{2}{|c|}{ Marca C } & \multicolumn{2}{|c|}{ Marca D } \\
\hline & $\mathrm{N}^{\mathrm{o}}$ & $\%$ & $\mathrm{~N}^{\mathrm{o}}$ & $\%$ & $\mathrm{~N}^{\mathrm{o}}$ & $\%$ & $\mathrm{~N}^{\mathrm{o}}$ & $\%$ \\
\hline Fragmentos & 9 & 90 & 9 & 90 & 8 & 80 & 10 & 100 \\
\hline de Insetos & 1 & 10 & 1 & 10 & 2 & 20 & 0 & 0 \\
\hline TOTAL & 10 & 100 & 10 & 100 & 10 & 100 & 10 & 100 \\
\hline Insetos & 10 & 100 & 10 & 100 & 10 & 100 & 10 & 100 \\
\hline mortos & 0 & 0 & 0 & 0 & 0 & 0 & 0 & 0 \\
\hline TOTAL & 10 & 100 & 10 & 100 & 10 & 100 & 10 & 100 \\
\hline \multirow[t]{2}{*}{ Ácaros } & 9 & 90 & 10 & 100 & 10 & 100 & 10 & 100 \\
\hline & 1 & 10 & 0 & 0 & 0 & 0 & 0 & 0 \\
\hline TOTAL & 10 & 100 & 10 & 100 & 10 & 100 & 10 & 100 \\
\hline Pêlos de & 4 & 40 & 3 & 30 & 2 & 20 & 2 & 20 \\
\hline Roedor & 6 & 60 & 7 & 70 & 8 & 80 & 8 & 80 \\
\hline TOTAL & 10 & 100 & 10 & 100 & 10 & 100 & 10 & 100 \\
\hline $\begin{array}{l}\mathrm{N}^{\circ} \text { médio de fragmentos de } \\
\text { insetos em } 50 \mathrm{~g} \text { de farinha }\end{array}$ & \multicolumn{2}{|c|}{$141,0 \pm 36,6$} & \multicolumn{2}{|c|}{$110,4 \pm 22,4$} & \multicolumn{2}{|c|}{$159,2 \pm 33,3$} & \multicolumn{2}{|c|}{$102,7 \pm 20,4$} \\
\hline Intervalo de variação & \multicolumn{2}{|c|}{29 a 533} & \multicolumn{2}{|c|}{39 а 296} & \multicolumn{2}{|c|}{33 a 485} & \multicolumn{2}{|c|}{24 a 291} \\
\hline
\end{tabular}


TABELA 2 - NÍVEL DE APROVAÇÃO E CONDENAÇÃO DE AMOSTRAS DE MACARRÃO, PRODUZIDAS E COMERCIALIZADAS POR QUATRO INDÚSTRIAS DE PASTIFÍCIO, DO ESTADO DO PARANÁ, ANALISADAS ATRAVÉS DO MÉTODO DA HIDRÓLISE ÁCIDA, COM REFERÊNCIA À LEGISLAÇÃO EM VIGOR

\begin{tabular}{lcccccccc}
\hline \multirow{2}{*}{ AMOSTRAS } & \multicolumn{2}{l}{ MARCA A } & \multicolumn{2}{l}{ MARCA B } & \multicolumn{2}{l}{ MARCA C } & \multicolumn{2}{l}{ MARCA D } \\
\cline { 2 - 9 } & $\mathrm{N}^{\circ}$ & $\%$ & $\mathrm{~N}^{\circ}$ & $\%$ & $\mathrm{~N}^{\circ}$ & $\%$ & $\mathrm{~N}^{\circ}$ & $\%$ \\
\hline Condenadas & 1 & 10 & 1 & 10 & 2 & 20 & 0 & 0 \\
Aprovadas & 9 & 90 & 9 & 9 & 8 & 80 & 10 & 100 \\
\hline TOTAL & 10 & 100 & 10 & 100 & 10 & 100 & 10 & 100 \\
\hline
\end{tabular}

Utilizando como parâmetro somente o número de fragmentos de insetos verifica-se que das 40 amostras de macarrão analisadas, apenas $10 \%$ estariam condenadas por apresentarem mais de 225 fragmentos de insetos em $225 \mathrm{~g}$ da amostra. Considerando, também, a presença de ácaros e de pêlos de roedor, observa-se que 0 índice de condenação aumenta, consideravelmente $(77,5 \%)$, sendo portanto, a contaminação por pêlos de roedor responsável por $67,5 \%$ da condenação.

Em duas das marcas de macarrão analisadas (A e B), os números máximos de fragmentos encontrados (Tabela 1), foram superiores a 317 e 342 obtidos por GECAN \& ATKINSON (4) e aos encontrados por ZAMBONI \& ATUI (7), ou seja, 312, 345 e 282.

Nas análises realizadas por GECAN \& ATKINSON (4) foi observada a presença de insetos mortos e/ou partes equivalentes, e de larvas em 2, 4 e 3\% das amostras analisadas. Para BONAFACCIA et al. (3) estes tipos de contaminantes pertencem a categoria "vários". ZAMBONI \& ATUI (7) também observaram que 4, 2 e 8\% do total de amostras analisadas apresentaram contaminação por insetos mortos e por larvas mortas de insetos. Tal fato poderia evidenciar contaminação da matéria-prima pós-moagem, em função da manipulação ou estocagem da farinha em condições higiênicas não satisfatórias e/ou da contaminação durante a elaboração e/ou na estocagem do macarrão. Na presente pesquisa não foi constatado este tipo de contaminação, demonstrando que a mesma não ocorreu pós-moagem. Além disso, os resultados obtidos em relação ao tamanho médio dos fragmentos recuperados, $0,23 \pm 0,02 \mathrm{~mm}$ com intervalo de variação de 0,06 a $0,58 \mathrm{~mm}$, mostraram-se inferiores ao tamanho dos fragmentos de insetos considerados pela HEALTH PROTECTION BRANCH (5), como provenientes de contaminação pós-moagem $(<0,595)$. Justifica-se assim a hipótese de que a contaminação ocorreu na matériaprima, isto é, a infestação interna já estava presente nos grãos de trigo, antes da moagem. 
o número máximo de ácaros mortos encontrados resume-se a dois, em apenas uma amostra, resultado inferior ao registrado por ZAMBONI \& ATUI(7), os quais constataram presença de ácaros mortos em quatro amostras.

Quanto aos pêlos de roedor $72,50 \%$ do total das amostras de macarrão apresentaram este tipo de sujidade, com intervalo de variação de 0 a 9 pêlos por amostra, superior portanto, à porcentagem observada por GECAN \& ATKINSON (4) (22 e 36\%), e por ZAMBONI \& ATUI (7) (35\%).

\section{CONCLUSÃO}

Dez por cento das amostras analisadas encontraram-se em desacordo com a legislação em vigor, por apresentarem mais de 225 fragmentos de insetos em $225 \mathrm{~g}$ de amostra.

A presença de pêlos de roedor foi o principal fator adicional de contaminação, sendo estas sujidades responsáveis por $67,5 \%$ da condenação.

Considerando ambas as sujidades (fragmentos de insetos e pêlos de roedor), verificou-se que $77,5 \%$ do total de amostras analisadas estavam em desacordo com a legislação vigente, tendo sido encontrados dois ácaros mortos, em apenas uma amostra.

Conclui-se que a contaminação deste produto decorreu da utilização de matéria-prima (farinha de trigo) contaminada.

Sugere-se às indústrias de alimentos que produzem massas o controle da matéria-prima, do maquinário utilizado, das instalações e o controle global do produto final para limitar a possibilidade de contaminação, ou seja, o cumprimento dos bons princípios de manufaturação "Good Manufacturing Practices" (G.M.P.) com o objetivo de oferecer produtos em ótimas condições de qualidade.

\section{Abstract}

Samples of wheat macaroni were examinated for sanitary quality. For extration of the light filth the acid hidrolysis method of the official Methods of Analysis of the Association of Analytical Chemists, was used. The percentage of wheat macaroni samples rejected was $10 \%$ due to the number of insect fragments exceeding the tolerance level (225 insect fragments in $225 \mathrm{~g}$ of sample). The contamination by rodent hairs was the main additional factor to reject the samples of the wheat macaroni. When this light filth, was considerated, the percentage of samples rejected increased to $77,5 \%$. 


\section{REFERÊNCIAS BIBLIOGRÁFICAS}

1 ASSOCIATION OF OFFICIAL ANALYTICAL CHEMISTS. Official Methods of Analysis of the Association of Analytical Chemists. 15. ed. Washington, DC., 1990. p. 369-406

2 BRASIL. Ministério da Saúde. Divisão Nacional de Vigilância Sanitária de Alimentos. Portaria $n^{\circ} 74$, de 4 de agosto de 1994. Diário oficial [da República Federativa do Brasil], Brasilia, 5 de agosto de 1994. Seção I, p. 11809

3 BONIFACCIA, G. , CUBADDA, R., GALLI, V. , \& PASQUI, L.A. Indagine sulle impurità solide (filth test) in paste di semola di grano duro di produzione nazionale. Nota 1. Técnica Molitora, v. 38, n. 3, p. 180-201, 1987.

4 GECAN, J.S. \& ATKINSON, J.C. Microanalytical quality of acaroni and noodles. J. Food Protec., v. 48, n. 5, p. 400-2, 1985 .

5 HEALTH PROTECTION BRANCH. Guidelines for extraneous material in food. Canadá : Health and Welfare, 1984. 5 p.

6 LANDIM, M.A. Levantamento das condições higiênicas da farinha de trigo e de massas alimentícias tipo espaguete. Viçosa, 1990. 57 p. Dissertação (Mestrado) - Universidade Federal de Viçosa, 1990.

7 ZAMBONI, C.Q. \& ATUI, M.B. Comparação entre métodos para pesquisa de sujidades e verificação das condições de higiene das massas alimentícias por microscopia. Rev. Inst. Adolfo Lutz, V. 49, n. 1, p. 11-17, 1989. 